\title{
Analysis of a Lensed Coreless Fiber by a Hybrid Technique Combining FD-BPM and FD-TDM
}

\author{
Junji Yamauchi, Member, IEEE, Koji Nishio, and Hisamatsu Nakano, Fellow, IEEE
}

\begin{abstract}
A technique connecting a scalar wide-angle finitedifference beam-propagation method(FD-BPM) with a scalar finite-difference time-domain method (FD-TDM) is systematically described and used to analyze a lensed coreless fiber. The propagating field and its phase distribution are calculated to demonstrate the phase adjustment effect of the lens. The power concentration due to the lens is evaluated by the directive gain used in the antenna engineering. The effect of an antireflection coating on the reflected power is also evaluated.
\end{abstract}

Index Terms - Finite-difference method, lensed fiber, optical beam propagation.

\section{INTRODUCTION}

M ANY numerical methods have been developed for the analysis of optical waveguides. Of them the finitedifference beam-propagation method (FD-BPM) [1] and the finite-difference time-domain method (FD-TDM) [2] are powerful tools of analyzing the propagating beam in optical waveguides. The FD-BPM based on the implicit scheme has the advantage of unconditional stability which allows the use of a large sampling grid and a transparent boundary condition [3]. Since the FD-BPM assumes only forward traveling waves, reflected waves generated at longitudinal discontinuities have been neglected. For the case in which the reflection cannot be neglected, the bidirectional BPM has been developed [4], [5].

On the other hand, the FD-TDM that directly solves Maxwell's equations can be applied to the analysis of the propagation, reflection, scattering, and radiation of electromagnetic waves in optical devices [6]. The result of the FD-TDM is accurate since no approximation is introduced with respect to polarization and propagating beam direction, but it requires great computation time and memories for the simulation of optically large structures.

Recently, a scalar FD-TDM was formulated in rectangular coordinates [7] and was extended to cylindrical coordinates [8], [9]. Compared with the vector FD-TDM, the scalar FDTDM requires less memory and is computationally more efficient. It should be noted, however, that the scalar FD-TDM still imposes a computational burden for large structures. From this viewpoint, a hybrid BPM-FDTD simulation has begun to attract attention [10]-[12].

In this paper, we systematically describe the hybrid technique that combines a scalar wide-angle FD-BPM and a scalar FD-TDM, and apply it to the analysis of a lensed coreless fiber

Manuscript received April 14, 1997; revised November 10, 1997

The authors are with the College of Engineering, Hosei University, Tokyo 184 Japan.

Publisher Item Identifier S 0733-8724(98)01886-6.
(CF) [13]-[15]. After confirming the validity of the present technique, we analyze the propagating field in the CF. Special attention is paid to focusing properties of the lensed CF. The far-field pattern is evaluated from the field at the endface. Furthermore, we reveal the effects of an antireflection (AR) coating [16] on the fields in the lens.

\section{Numerical MethoD}

\section{A. Formulation}

We consider the propagation of a circularly symmetric field $E(r, z)$ in an optical fiber, and derive the equations for the FDBPM. The scalar Helmholtz equation in cylindrical coordinates is

$$
\frac{\partial^{2} E}{\partial z^{2}}+\frac{\partial^{2} E}{\partial r^{2}}+\frac{1}{r} \frac{\partial E}{\partial r}+k_{0}^{2} n^{2}(r, z) E=0
$$

where $k_{0}$ is a wavenumber in free space, $E(r, z) \exp (j \omega t)$ is a component of the electric field at an angular frequency of $\omega$, and $n(r, z)$ is the index profile of the waveguide. For convenience, the electric field $E$ is expressed as

$$
E(r, z)=\psi(r, z) \exp \left(-j k_{0} n_{0} z\right)
$$

where $n_{0}$ is the appropriately chosen reference refractive index. In this paper, the reference refractive index $n_{0}$ is chosen to be that in the cladding. Substituting (2) into (1), we obtain

$$
-\frac{\partial^{2} \psi}{\partial z^{2}}+2 j k_{0} n_{0} \frac{\partial \psi}{\partial z}=\left(\frac{\partial^{2}}{\partial r^{2}}+\frac{1}{r} \frac{\partial}{\partial r}+k_{0}^{2}\left(n^{2}-n_{0}^{2}\right)\right) \psi .
$$

When we neglect the second derivative with respect to $z$, (3) reduces to the paraxial equation. In this paper, we use the $(1,1)$ Padé approximant operator [17], so that we derive

$$
\frac{\partial \psi}{\partial z}=-\frac{\frac{j P}{2 k_{0} n_{0}}}{1+\frac{P}{4 k_{0}^{2} n_{0}^{2}}} \psi
$$

where

$$
P=\frac{\partial^{2}}{\partial r^{2}}+\frac{1}{r} \frac{\partial}{\partial r}+k_{0}^{2}\left(n^{2}-n_{0}^{2}\right)
$$

We apply the Crank-Nicholson scheme to (4), and derive the difference equations. Using the simplifying symbol

$$
\psi(r, z)=\psi((i-1) \Delta r, k \Delta z)=\psi_{i}^{k}
$$

where $i$ and $k$ are integers, we obtain the following difference equation for $i>1$

$$
\begin{aligned}
& a^{+} \psi_{i-1}^{k+1}+(\chi+\zeta) \psi_{i}^{k+1}+a^{-} \psi_{i+1}^{k+1} \\
& \quad=\xi a^{+} \psi_{i-1}^{k}+(\chi+\xi \zeta) \psi_{i}^{k}+\xi a^{-} \psi_{i+1}^{k}
\end{aligned}
$$




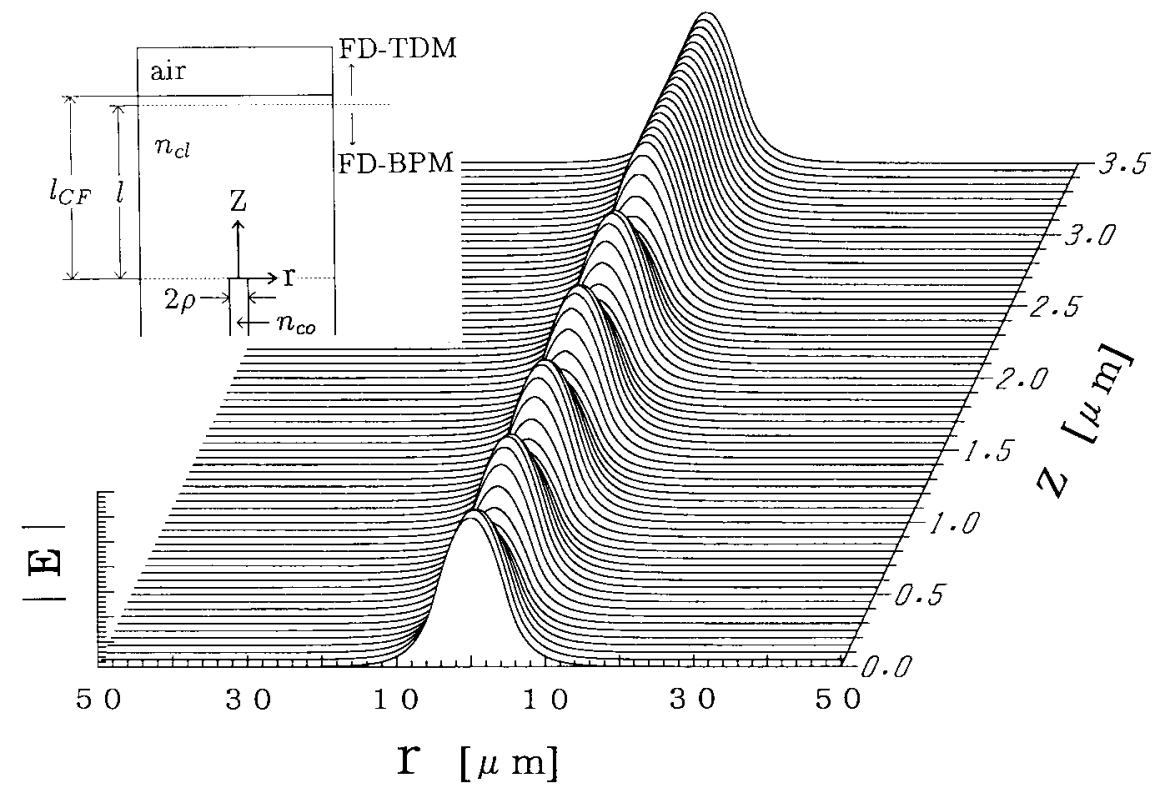

Fig. 1. Field distribution in the coreless fiber.

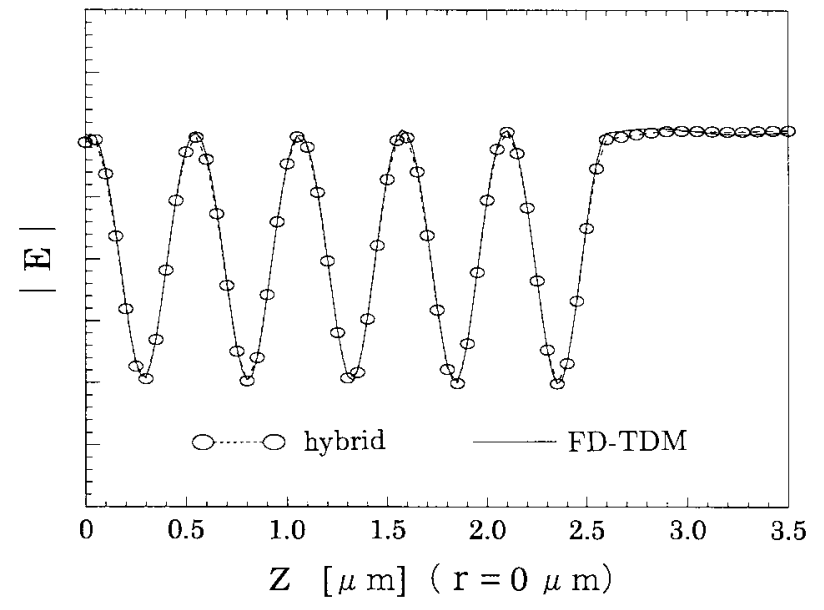

Fig. 2. Comparison between the propagating fields obtained from the hybrid technique and the FD-TDM $(r=0 \mu \mathrm{m})$.

where $a^{ \pm}=1 \mp 1 / 2(i-1), \chi=\left(4 k_{0}^{2} n_{0}^{2} \Delta r^{2}\right) /(1+$ $\left.j k_{0} n_{0} \Delta z\right), \zeta=\Delta r^{2} k_{0}^{2}\left(n^{2}-n_{0}^{2}\right)-2$ and $\xi=(1-$ $\left.j k_{0} n_{0} \Delta z\right) /\left(1+j k_{0} n_{0} \Delta z\right)$.

The indeterminate form at the origin $r=0$ can be evaluated by L'Hospital's rule. Taking into account the circular symmetry of the field, $\psi_{0}^{k}=\psi_{2}^{k}$, we obtain for $i=1$

$$
(\chi+\eta) \psi_{1}^{k+1}+4 \psi_{2}^{k+1}=(\chi+\xi \eta) \psi_{1}^{k}+4 \xi \psi_{2}^{k}
$$

where $\eta=\Delta r^{2} k_{0}^{2}\left(n^{2}-n_{0}^{2}\right)-4$.

Equations (7) and (8) can be easily solved by efficient techniques such as the Thomas algorithm. The transparent boundary condition developed by Hadley [3] is imposed at the edge of the calculation region.

We, next, describe the scalar FD-TDM for circularly symmetric waveguides. The electric field $\mathcal{E}(r, z, t)$ satisfies the

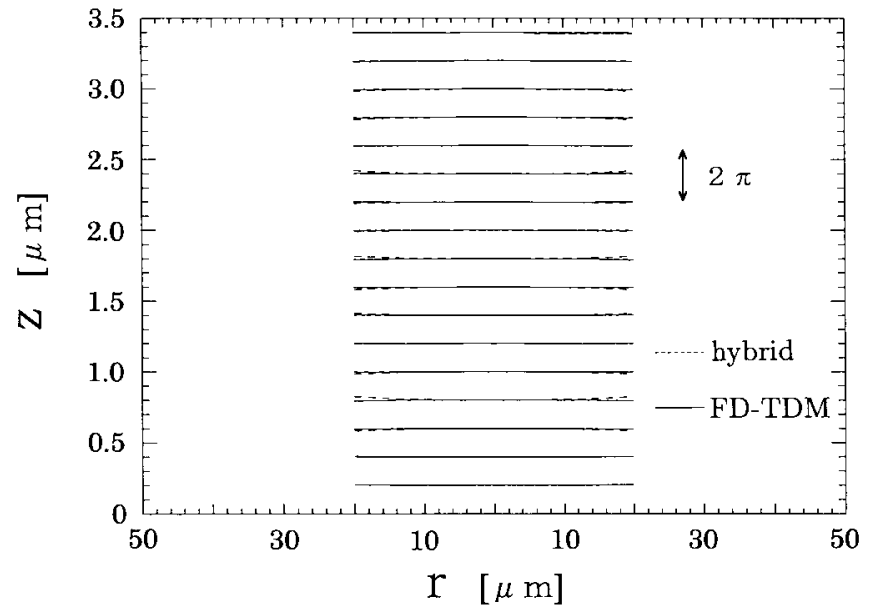

Fig. 3. Comparison between the phase distributions obtained from the hybrid technique and the FD-TDM.

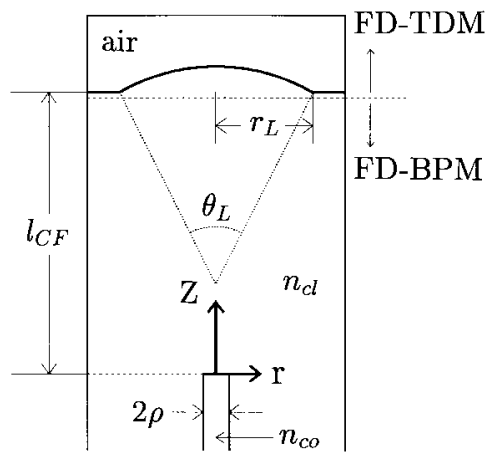

Fig. 4. Configuration of a hemispherically ended coreless fiber.

scalar wave equation

$$
\frac{\partial^{2} \mathcal{E}}{\partial r^{2}}+\frac{1}{r} \frac{\partial \mathcal{E}}{\partial r}+\frac{\partial^{2} \mathcal{E}}{\partial z^{2}}-\frac{n^{2}}{c^{2}} \frac{\partial^{2} \mathcal{E}}{\partial t^{2}}=0
$$


where $c$ is the velocity of light in free space. Using a central difference formula, we derive

$$
\begin{aligned}
\mathcal{E}_{i}^{k}(m+1)= & 2\left[1-\left(\frac{c \Delta t}{n \Delta r}\right)^{2}-\left(\frac{c \Delta t}{n \Delta z}\right)^{2}\right] \\
& \cdot \mathcal{E}_{i}^{k}(m)-\mathcal{E}_{i}^{k}(m-1) \\
& +\left(\frac{c \Delta t}{n \Delta r}\right)^{2}\left[\left\{1+\frac{1}{2(i-1)}\right\} \mathcal{E}_{i+1}^{k}(m)\right. \\
& \left.+\left\{1-\frac{1}{2(i-1)}\right\} \mathcal{E}_{i-1}^{k}(m)\right] \\
& +\left(\frac{c \Delta t}{n \Delta z}\right)^{2}\left[\mathcal{E}_{i}^{k+1}(m)+\mathcal{E}_{i}^{k-1}(m)\right]
\end{aligned}
$$

where $n=n_{i}^{k}, t=(m-1) \Delta t, r=(i-1) \Delta r$, and $z=(k-$ 1) $\Delta z$, in which $\Delta t, \Delta r$ and $\Delta z$ represent increments along the time, transverse and propagation directions, respectively.

On the fiber axis, $r=0$, we again apply L'Hospital's rule to the second term of (9). Finally, we have

$$
\begin{aligned}
\mathcal{E}_{1}^{k}(m+1)= & 2\left[1-2\left(\frac{c \Delta t}{n \Delta r}\right)^{2}-\left(\frac{c \Delta t}{n \Delta z}\right)^{2}\right] \\
& \cdot \mathcal{E}_{1}^{k}(m)-\mathcal{E}_{1}^{k}(m-1) \\
& +4\left(\frac{c \Delta t}{n \Delta r}\right)^{2} \mathcal{E}_{2}^{k}(m)+\left(\frac{c \Delta t}{n \Delta z}\right)^{2} \\
& \cdot\left[\mathcal{E}_{1}^{k+1}(m)+\mathcal{E}_{1}^{k-1}(m)\right]
\end{aligned}
$$

where $n=n_{1}^{k}$. In contrast to the FD-BPM, the FD-TDM is an explicit scheme in which the field values at two previous time steps are required. At the edge of the calculation region, we apply Mur's absorbing boundary condition [18] extended to cylindrical coordinates [9].

\section{B. Technique of Connecting FD-BPM with FD-TDM}

In this section, we describe the method of utilizing the field obtained from the FD-BPM as the incident field in the FD-TDM. First, the forward-propagating wave is calculated using a wide-angle FD-BPM of (7) and (8). Although the paraxial FD-BPM can also be used, the wide-angle FD-BPM is more appropriate when the fields contain radiation modes. The obtained electric field $E(r, z)$ at a position $(z=l)$ where the FD-BPM is connected with the FD-TDM, is expressed as

$$
E(r, l)=\psi(r, l) \exp \left(-j k_{0} n_{0} l\right) \text {. }
$$

Separating the electric field into an amplitude term $\bar{E}(r, l)$ and a phase term $\varphi(r, l)$, we can rewrite (12) as

$$
E(r, l)=\bar{E}(r, l) \exp [j \varphi(r, l)] .
$$

On the other hand, the electric field for the scalar FD-TDM can be expressed as

$$
\mathcal{E}(r, l, t)=E(r, l) \exp (j \omega t) .
$$

Substituting (13) into (14), and changing it into the expression of an instantaneous value, we can get the incident field of the FD-TDM located at the connecting position. As revealed in [12], the existence of the phase term $\varphi$ gives rise to considerable parasitic waves. Therefore, we apply a gradual exicitation method [19], [20], in which a sine function of the quarter period is multiplied. As a result, we use

$$
\begin{gathered}
\mathcal{E}(r, l, t)=\bar{E}(r, l) \sin \left[k_{0} c t+\varphi(r, l)\right] \sin (\pi c t / 2 \Delta \tau) \\
\text { for } c t \leq \Delta \tau \\
\mathcal{E}(r, l, t)=\bar{E}(r, l) \sin \left[k_{0} c t+\varphi(r, l)\right] \\
\quad \text { for } c t>\Delta \tau
\end{gathered}
$$

where $\Delta \tau / c$ is a rising time. As discussed in [12], the use of (15) greatly contributes to reduction of the parasitic waves with subsequent faster convergence of the numerical results. In this paper, we choose $\Delta \tau=9.3 \mu \mathrm{m}$.

Once a steady-state field is obtained in the FD-TDM region, the backward-propagating wave is extracted and is used as the incident field for the backward FD-BPM. The steady-state field in the FD-BPM region can be obtained by adding the forward- and backward-propagating waves [4]. Without loss of generality, the present technique can also be used in Cartesian coordinates.

\section{Confirmation of the Connecting Technique}

To confirm the validity of the hybrid technique, we compare the field obtained from the hybrid technique with that obtained when only the FD-TDM is employed. Consideration is given to a coreless fiber (CF) with a facet shown in the inset of Fig. 1. The length of the CF is taken to be $l_{\mathrm{CF}}=2.6 \mu \mathrm{m}$. The adjacent media to the $\mathrm{CF}$ is air. The core radius of the input fiber is $\rho=5 \mu \mathrm{m}$, and the refractive indices of core and cladding are $n_{\mathrm{co}}=1.504$ and $n_{\mathrm{cl}}=1.50$, respectively. A wavelength of $\lambda=1.55 \mu \mathrm{m}$ is used, so that the normalized frequency is $V \simeq 2.22$. The input field is the fundamental mode $L P_{01}$ which is launched at $z=0 \mu \mathrm{m}$. The connecting position between the FD-BPM and the FD-TDM is chosen to be $l=2.5 \mu \mathrm{m}$, so that the incident field for the FDTDM is launched at $z=2.5 \mu \mathrm{m}$ toward the $+z$ direction using the technique described in [9] and [21]. The computation parameters are taken to be $\Delta r=\Delta z=\rho / 200=0.025 \mu \mathrm{m}$ and $c \Delta t=0.0031 \mu \mathrm{m}$ in the FD-TDM region. The same discretization mesh is also used for the FD-BPM region. The number of transverse grid points is 2000 .

Fig. 1 shows the field distribution calculated from the hybrid technique. Almost the same distribution is obtained when only the FD-TDM is employed. Due to the reflection at the interface with air, the field exhibits a standing wave in the CF. Fig. 2 shows a comparison between the fields obtained from the hybrid technique and the FD-TDM. The field is compared at $r=0 \mu \mathrm{m}$. It is found that the two curves are almost indistinguishable. Slight field variation observed in the air region is due to incompleteness of the absorbing boundary condition. The phase variation obtained from the hybrid technique also agrees well with that obtained from the FD-TDM, as shown in Fig. 3. The phase difference is observed only in negligible fields. From the above-mentioned results, it can be said that the field obtained from the FD-BPM is satisfactorily connected with that in the FD-TDM.

The FD-BPM and the FD-TDM have different numerical dispersion characteristics. Hence, we also check the convergence of the numerical results as a function of mesh sizes $\Delta r$ and $\Delta z$. Further calculation shows that the results 


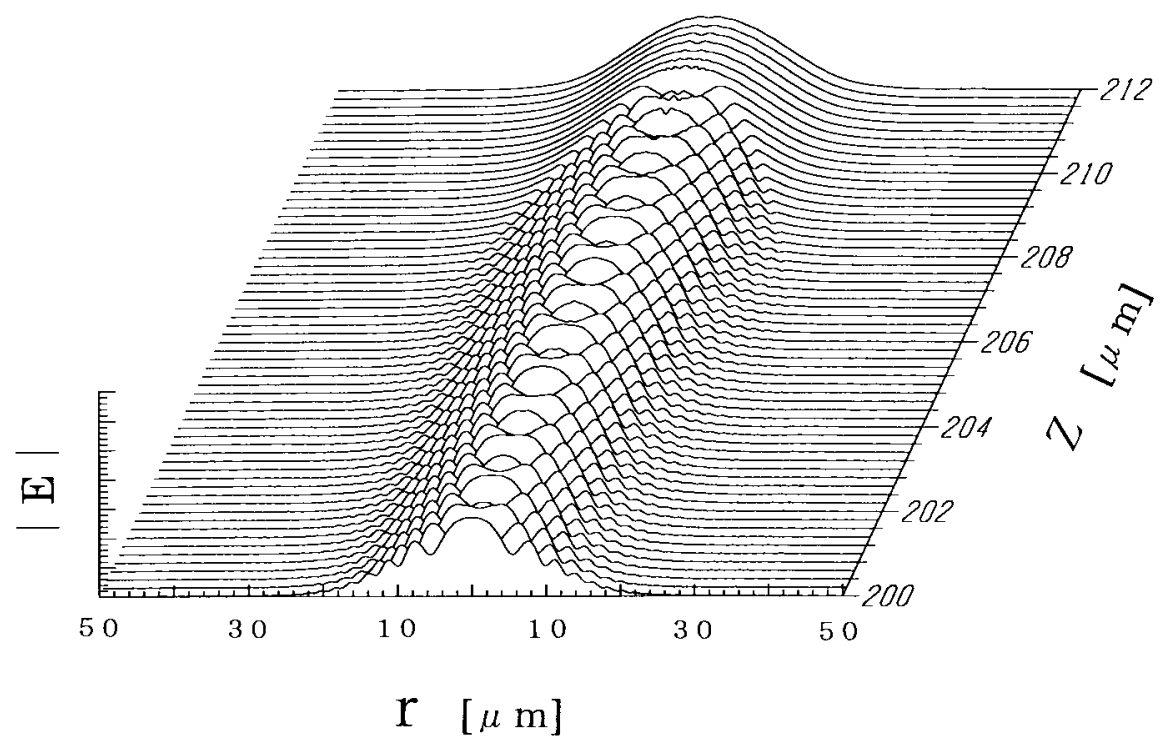

(a)

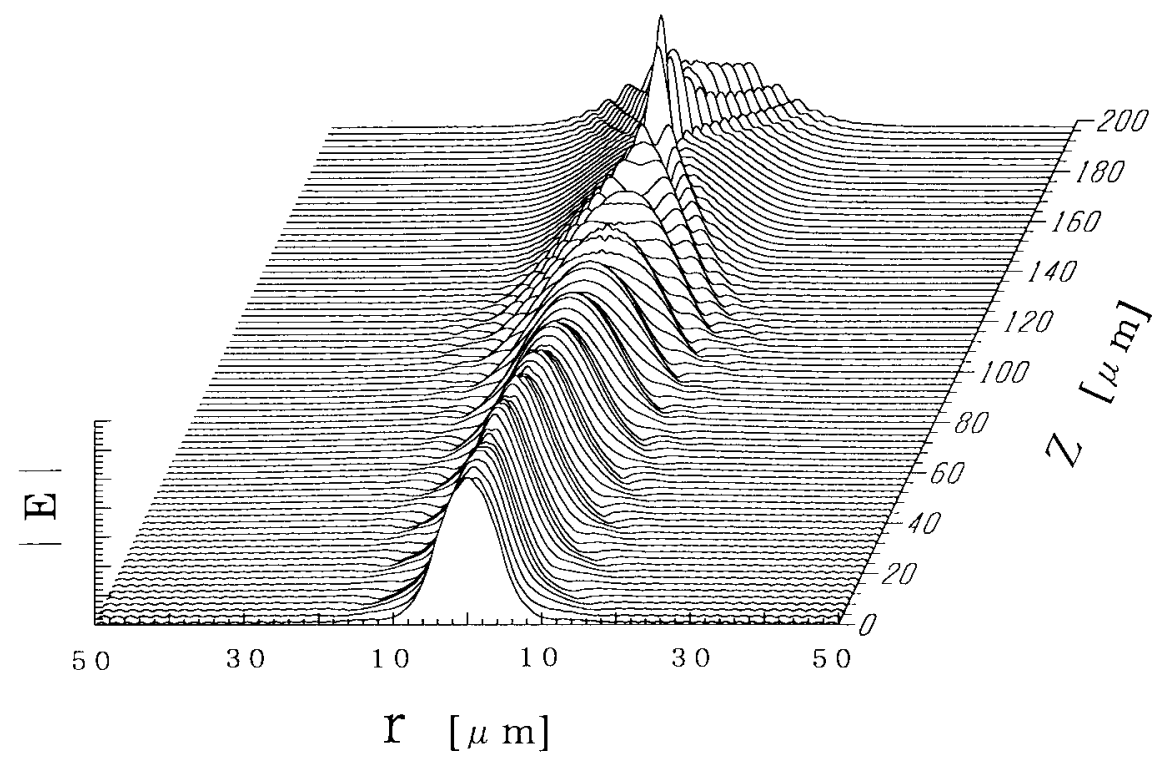

(b)

Fig. 5. Field distributions.

obtained with $\Delta r=\Delta z=\rho / 100=0.05 \mu \mathrm{m}$ are virtually superimposed on the results in Figs. 1, 2 and 3.

\section{ANALysis of A Lensed CoReless Fiber}

We now analyze a hemispherically-ended coreless fiber (CF) shown in Fig. 4. The CF is identical to that in Fig. 1 with the exception of a CF length of $l_{\mathrm{CF}}=200 \mu \mathrm{m}$. The hemispherical lens tipped on the $\mathrm{CF}$ end surface is formed by the adjustment of a lens radius $r_{\mathrm{L}}$ and a center angle $\theta_{\mathrm{L}}$ (A stepped approximation is used for the curved endface). The refractive index of the lens is the same as that of the cladding. A numerical calculation is carried out for $\Delta r=$ $\Delta z=\rho / 200=0.025 \mu \mathrm{m}$ and $c \Delta t=0.0031 \mu \mathrm{m}$. The position where the FD-BPM is connected with the FD-TDM is taken to be $l=199.9 \mu \mathrm{m}$.
Fig. 5 shows the field distributions $(c t=62 \mu \mathrm{m})$ with $r_{\mathrm{L}}=40 \mu \mathrm{m}$ and $\theta_{\mathrm{L}}=60^{\circ}$. These configuration parameters can be regarded as being optimum as will be shown in Fig. 7 . Note that the scale of the propagating direction $z$ in the lens [Fig. 5(a)] is expanded, compared with that in the $\mathrm{CF}$ [Fig. 5(b)]. We can observe the complicated field distribution in the lens. This is due to the reflected waves caused at the lens endface with air. The reflected power of the fundamental mode is calculated to be $4.2 \%$, which is the same as a value evaluated from the simple Fresnel coefficient. The reflected wave generated at the lens endface propagates in the $\mathrm{CF}$, causing interference with the forward-propagating wave. We can observe a peak amplitude near a propagation distance of $150 \mu \mathrm{m}$.

The phase variation corresponding to Fig. 5 is presented in Fig. 6. Fig. 6(b) shows that the constant phase plane is 


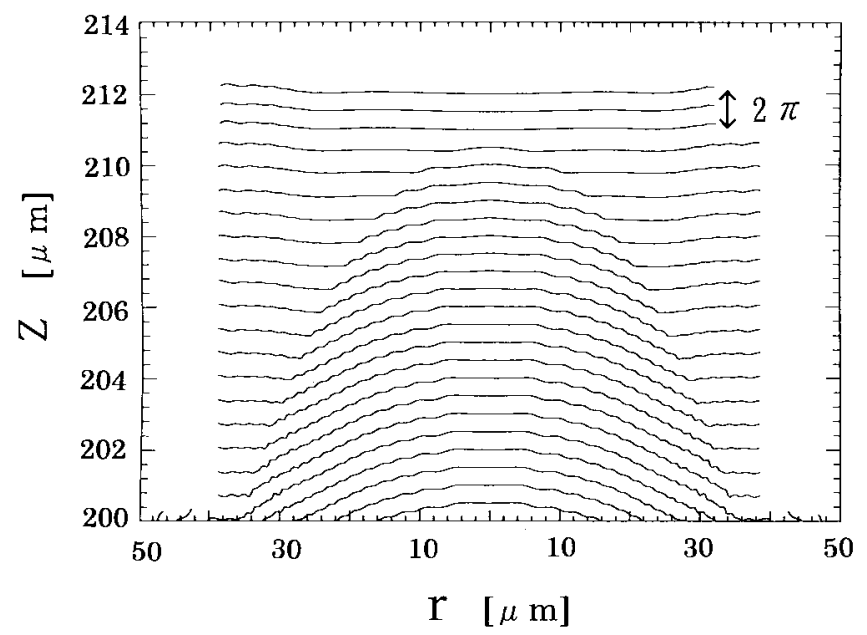

(a)

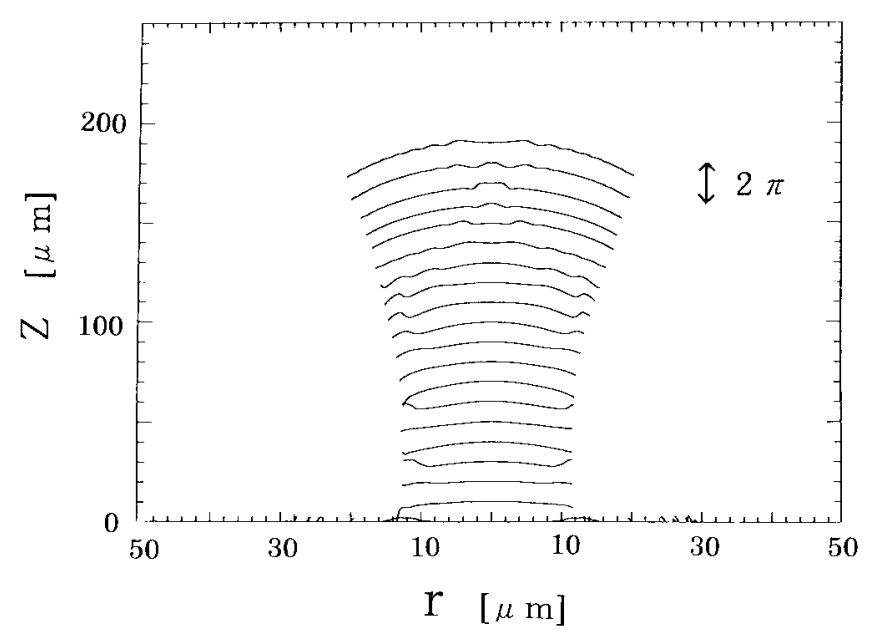

(b)

Fig. 6. Phase distributions.

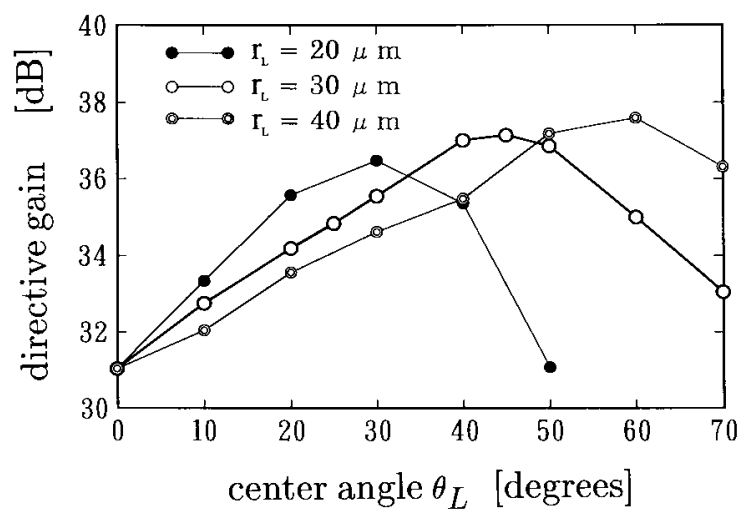

Fig. 7. Directive gain as a function of center angle.

gradually distorted with propagation, while Fig. 6(a) shows that the distorted phase variation is gradually recovered. This is due to the fact that the local velocity of the wave in air is higher than that in the lens. It is demonstrated that the lens operates as a phase adjustment element.

To investigate the focusing effect of the hemispherical lens, we evaluate the far-field pattern, using the field $E(r)$ in air.

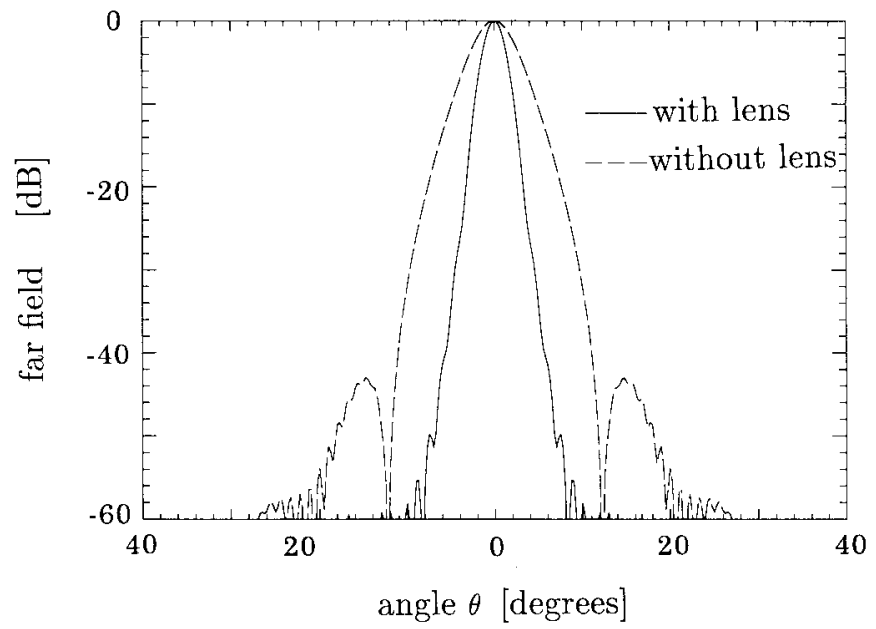

Fig. 8. Comparison in the far-field pattern.

According to the Kirchhoff-Huygens diffraction integral, the far field $E(R, \theta)$ is given as follows:

$$
E(R, \theta)=\frac{j \pi}{\lambda R} \exp \left(-j k_{0} R\right) D(\theta)
$$

in which $D(\theta)$ is defined as

$$
D(\theta)=(1+\cos \theta) \int_{0}^{r_{w}} E(r) J_{0}\left(k_{0} r \sin \theta\right) r d r
$$

where $J_{0}$ is the zeroth-order Bessel function of the first kind and $r_{w}$ is the computational window dimension. The integration with respect to $r$ is performed using the trapezoidal rule.

Introducing the directive gain often used in the antenna engineering is convenient to know how well the CF concentrates power into a limited solid angle. The directive gain can be defined as the ratio of the maximum radiation intensity to the average radiation intensity. The directive gain $G$ is expressed as

$$
\begin{aligned}
G & =10 \log _{10}\left[\frac{\frac{\left|E_{\max }\right|^{2}}{2 Z_{0}}}{\frac{P_{\text {in }}}{4 \pi R^{2}}}\right] \\
& =10 \log _{10}\left[\frac{2 \pi R^{2}\left|E_{\max }\right|^{2}}{Z_{0} P_{\text {in }}}\right] \quad[\mathrm{dB}]
\end{aligned}
$$

where $Z_{0}$ represents the characteristic impedance of free space and $P_{\text {in }}$ is the input power written as

$$
P_{\text {in }}=\frac{\pi n_{e}}{Z_{0}} \int_{0}^{r_{w}}\left|E_{\text {in }}(r)\right|^{2} r d r
$$

in which $E_{\mathrm{in}}(r)$ is the incident field and $n_{e}$ is the effective refractive index of the fundamental mode of the input fiber.

Substituting (17) and (20) into (19), we obtain

$$
G=10 \log _{10}\left[\frac{2 \pi^{2}\left|D_{\max }\right|^{2}}{\lambda^{2} n_{e} \int_{0}^{r_{w}}\left|E_{\mathrm{in}}(r)\right|^{2} r d r}\right] \quad[\mathrm{dB}] .
$$

The characteristics of the directive gain as a function of the center angle $\theta_{L}$ are shown in Fig. 7. In this Figure, the lens 


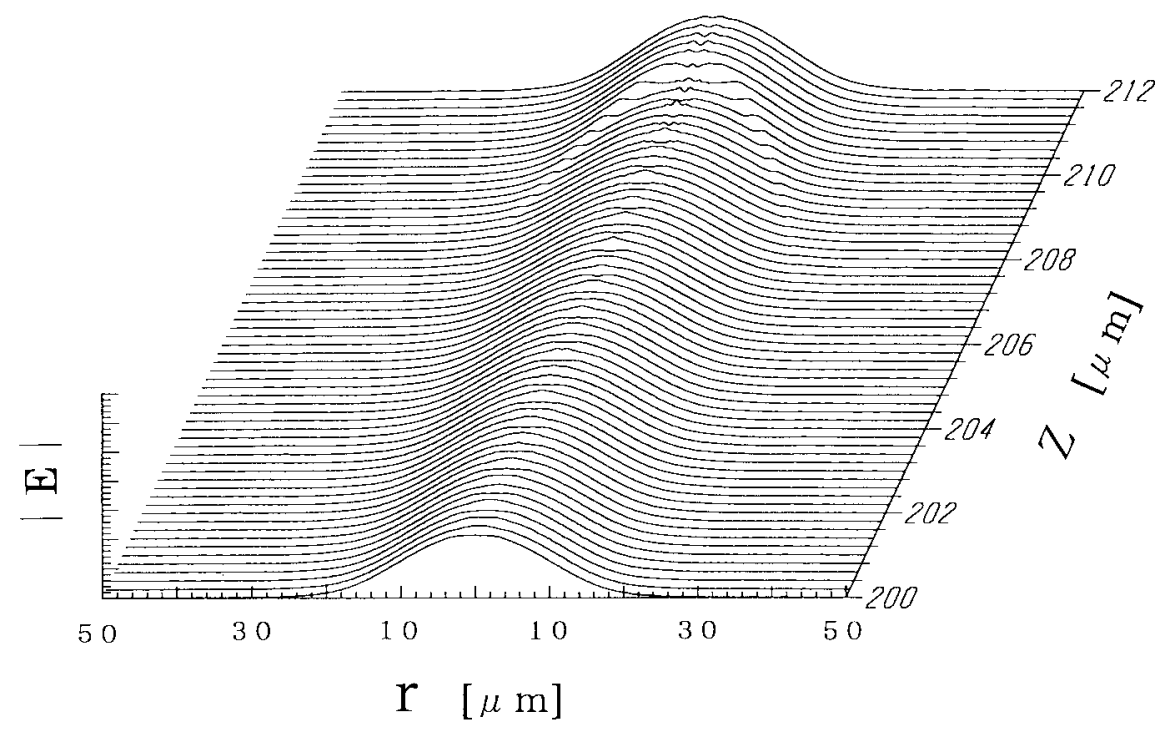

(a)

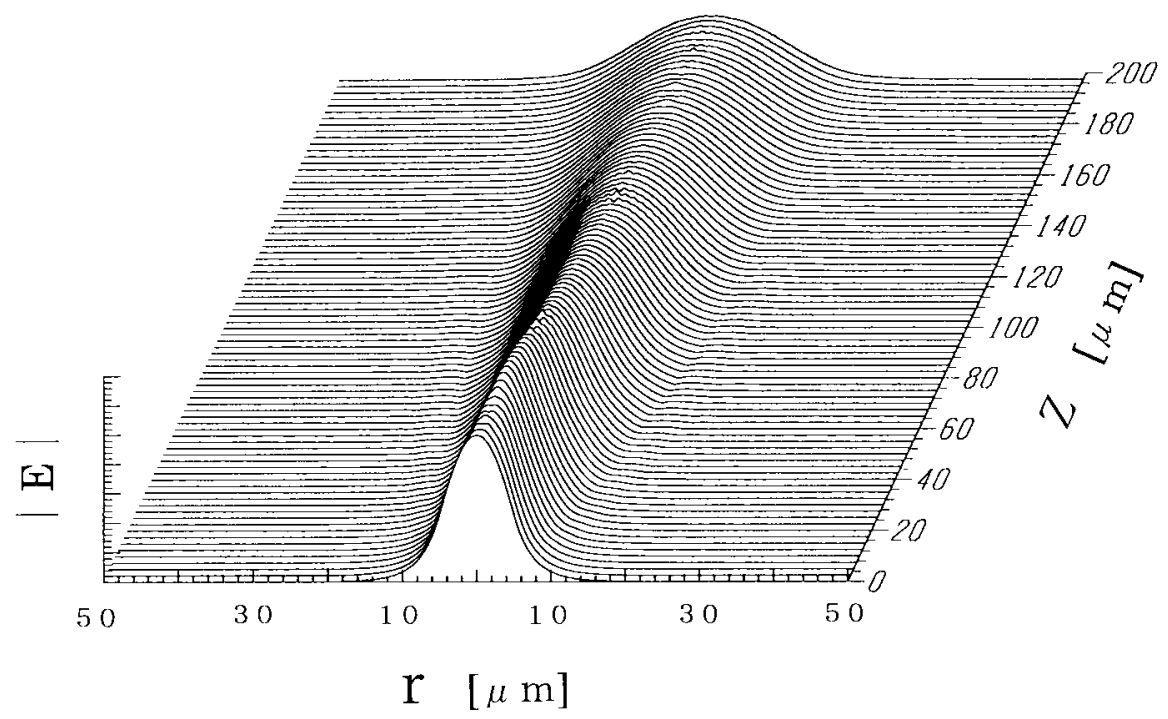

(b)

Fig. 9. Field distributions. The coreless fiber is coated with the AR layer $\left(n_{\mathrm{ac}}=1.225\right)$.

radius $r_{L}$ is used as a parameter and $\theta_{L}=0^{\circ}$ corresponds to the case without the lens. As can be seen, each curve indicates the existence of an optimum shape giving a maximum directive gain. Within the range of this analysis, the maximum directive gain is found to be $37.6 \mathrm{~dB}$ for $r_{L}=40 \mu \mathrm{m}$ and $\theta_{L}=60^{\circ}$, which is increased by $6.6 \mathrm{~dB}$ as compared to that without the lens.

Fig. 8 shows the far-field patterns radiated from the $\mathrm{CF}$ with the optimized lens $\left(r_{L}=40 \mu \mathrm{m}\right.$ and $\left.\theta_{L}=60^{\circ}\right)$ and from the $\mathrm{CF}$ without the lens. Each pattern is normalized by the maximum value. Due to the phase adjustment effect, the beam radiated from the lensed $\mathrm{CF}$ is sharper than that from the $\mathrm{CF}$ without the lens.

Finally, we treat a lensed CF with an AR coating. Taking account of impedance matching between the waveguide and air, the ideal refractive-index of the AR coating should be $n_{\mathrm{ac}}=\sqrt{n_{\mathrm{cl}}}$. Hence, the refractive index of the AR coating is fixed to be $n_{\mathrm{ac}}=1.225$, and the AR layer thickness is $\lambda_{\mathrm{g}} / 4=0.325 \mu \mathrm{m}$. The field distribution $(c t=62 \mu \mathrm{m})$ for the optimum lens coated with the AR layer is shown in Fig. 9. In contrast to Fig. 5(a), the standing wave is not observed in the lens. It is found that the reflected fields are successfully suppressed due to the AR coating, and the reflected power from the endface is reduced to approximately $0.01 \%$. Further calculation shows that the far-field pattern is almost the same as that observed without the AR coating.

Although the reflected fields are greatly suppressed by the ideal AR coating, the refractive index of the AR coating, $n_{\mathrm{ac}}=1.225$, chosen in the above analysis is not realistic. Therefore, we also analyze the case for $n_{\mathrm{ac}}=1.38$, which corresponds to $\mathrm{MgF}_{2}$. The reflected power is calculated to be $1.5 \%$.

\section{CONCLUSIONS}

We have systematically described a hybrid technique that combines the scalar FD-BPM and the scalar FD-TDM in cylin- 
drical coordinates. The hybrid technique enables us to analyze optically large structures with a longitudinal discontinuity and has the advantage of computational efficiency compared with the conventional technique in which only the FD-TDM is applied to the large structures.

As an application, a lensed coreless fiber has been analyzed. The phase adjustment effect of the lens is demonstrated by the phase distribution of the propagating field. The far-field is also calculated and the effect of power concentration due to the lens is evaluated by the directive gain often used in the antenna engineering. The parameters of an optimum lens are determined by the directive gain characteristics. Consideration is also given to the effect of an antireflection coating.

\section{ACKNOWLEDGMENT}

The authors would like to thank Dr. R. P. Ratowsky of Lawrence Livermore National Laboratory, University of California, who drew their attention to [10].

\section{REFERENCES}

[1] Y. Chung and N. Dagli, "An assessment of finite difference beam propagation method," IEEE J. Quantum Electron., vol. 26, pp. 1335-1339, Aug. 1990.

[2] K. S. Yee, "Numerical solution of initial boundary value problems involving Maxwell's equations in isotropic media," IEEE Trans. Antennas Propagat., vol. AP-14, pp. 302-307, Mar. 1966.

[3] G. R. Hadley, "Transparent boundary condition for beam propagation," Opt. Lett., vol. 16, no. 9, pp. 624-626, 1991.

[4] P. Kaczmarski and P. E. Lagasse, "Bidirectional beam propagation method," Electron. Lett., vol. 24, pp. 675-676, 1988.

[5] G. H. Jin, J. Harari, J. P. Vilcot, and D. Decoster, "Improved bidirectional beam propagation method for analysis of reflection on nonparallel interfaces," Electron. Lett., vol. 31, pp. 1867-1869, 1995.

[6] S. T. Chu and S. K. Chaudhuri, "A finite-difference time-domain method for the design and analysis of guided-wave optical structures," J. Lightwave Technol., vol. 7, pp. 2033-2038, Dec. 1989.

[7] W. P. Huang, S. T. Chu, A. Goss, and S. K. Chaudhuri, "A scalar finitedifference time-domain approach to guided-wave optics," IEEE Photon. Technol. Lett., vol. 3, no. 6, pp. 524-526, 1991

[8] J. Yamauchi, Y. Akimoto, M. Nibe, and H. Nakano, "Wide-angle propagating beam analysis for circularly symmetric waveguides: Comparison between FD-BPM and FD-TDM," IEEE Photon. Technol. Lett., vol. 8, pp. 236-238, Feb. 1996.

[9] J. Yamauchi, M. Nibe, and H. Nakano, "Scalar FD-TD method for circularly symmetric waveguides," Optic. Quantum Electron., vol. 29, no. 4, pp. 451-460, 1997.

[10] J. S. Kallman, "Hybrid 2-D BPM-FDTD simulation," Nat. Radio Sci. Meeting, vol. D/B1-2, p. 108, 1996.

[11] J. Yamauchi, K. Nishio, and H. Nakano, "Analysis of a lensed coreless fiber by a hybrid technique that involves FD-BPM and FD-TDM," Integrated Photon. Res., vol. IMH16-4, pp. 209-212, 1996.

[12] _ "Hybrid numerical technique combining the finite-difference beam-propagation method and the finite-difference time-domain method," Opt. Lett., vol. 22, no. 5, pp. 259-261, 1997.

[13] G. Wenke and Y. Zhu, "Comparison of efficiency and feedback characteristics of techniques for coupling semiconductor lasers to single-mode fiber," Appl. Opt., vol. 22, no. 23, pp. 3837-3844, 1983.

[14] J. Yamada, Y. Murakami, J. Sakai, and T. Kimura, "Characteristics of a hemispherical microlens for coupling between a semiconductor laser and single-mode fiber," IEEE J. Quantum Electron., vol. QE-16, pp. 1067-1072, Oct. 1980.

[15] K. Shiraishi, N. Oyama, K. Matsumura, I. Ohishi, and S. Suga, "A fiber lens with a long working distance for integrated coupling between laser diodes and single-mode fibers," J. Lightwave Technol., vol. 13, pp. 1736-1744, Aug. 1995.

[16] J. Yamauchi, M. Mita, S. Aoki, and H. Nakano, "Analysis of antireflection coatings using the FD-TD method with the PML absorbing boundary condition," IEEE Photon. Tech. Lett., vol. 8, no. 2, pp. 239-241, 1996.
[17] G. R. Hadley "Wide-angle beam propagation using Padé approximant operators," Opt. Lett., vol. 17, no. 20, pp. 1426-1428, 1992

[18] G. Mur, "Absorbing boundary conditions for the finite-difference approximation of the time-domain electromagnetic-field equations," IEEE Trans. Electromag. Compat., vol. EMC-23, pp. 377-382, 1981.

[19] S. M. Lee, W. C. Chew, S. L. Chuang, and J. J. Coleman, "Bending loss in optical waveguides for nonplanar laser array applications," J. Appl. Phys., vol. 71(6), no. 15, pp. 2513-2520, 1992.

[20] Y. Naka, H. Ikuno, A. Yata, and M. Nishimoto, "Numerical analysis of dielectric slab optical waveguides using the FD-TD method with a "Perfectly Matched Layer" absorbing boundary condition," in Asia Pacific Microwave Conf., vol. SO7-2, 1995.

[21] S. T. Chu, W. P. Huang, and S. K. Chaudhuri, "Simulation and analysis of waveguide based optical integrated circuits," Comput. Phys. Commun., vol. 68, pp. 451-484, 1991.

Junji Yamauchi (M'85) was born in Nagoya, Japan, on August 23, 1953. He received the B.E., M.E., and Dr.E. degrees from Hosei University, Tokyo, Japan, in 1976, 1978, and 1982, respectively.

From 1984 to 1988, he served as a Lecturer at the Electrical Engineering Department of Tokyo Metropolitan Technical College. Since 1988, he has been a member of the Faculty of Hosei University, where he is now a Professor of Electronic Informatics. His research interests include optical waveguides and circularly polarized antennas.

Dr. Yamauchi is a member of the Optical Society of America and the Institute of Electronics, Information and Communication Engineers (IEICE) of Japan.

Koji Nishio was born in Tokyo, Japan, on April 6, 1971. He received the B.E. and M.E. degrees from Hosei University, Tokyo, Japan, in 1995 and 1997, respectively.

He joined Dowa Mining Co., Ltd., Tokyo, in 1997.

Mr. Nishio is a member of the Institute of Electronics, Information and Communication Engineers (IEICE) of Japan.

Hisamatsu Nakano (M'75-SM'87-F'92) was born in Ibaraki, Japan, on April 13, 1945. He received the B.E., M.E., and Dr.E. degrees in electrical engineering from Hosei University, Tokyo, in 1968, 1970, and 1974, respectively.

Since 1973, he has been a member of the Faculty of Hosei University, where he is now a Professor of Electronic Informatics. His research topics include numerical methods for antennas, electromagnetic wave scattering problems, and lightwave problems. He has published more than 130 refereed journal papers and 90 international symposium papers on antenna and relevant problems. He is the author of the book Helical and Spiral Antennas (New York: Wiley Research Studies Press, 1987). In 1996, he published the chapter "Antenna analysis using integral equations," in the book Analysis Methods of Electromagnetic Wave Problems, (Dedham, MA: Artech House, vol. 2). He has developed a parabolic antenna using backfire helical feed for direct reception of broadcasting satellite TV programs (DBS). He has also developed two types of small indoor flat DBS antennas using novel elements: curled and extremely low-profile helical elements. His other developments include microstrip antennas for global positioning systems (GPS), personal handy telephone antennas, and small dual-polarization Cassegrain antennas for direct reception of communication satellite TV programs. He was a Visiting Associate Professor at Syracuse University, Syracuse, NY (May to September, 1981), where he worked on numerical analysis of electromagnetic coupling between wires and slots, a Visiting Professor at University of Manitoba, Canada (from March to September, 1986), researching numerical techniques for analysis of microstrip antennas, and a Visiting Professor at University of California, Los Angeles (from September 1986 to March 1987), working on microstrip line antenna analysis.

Dr. Nakano was the recipient of an International Scientific Exchange Award from the Natural Sciences and Engineering Research Council of Canada. In 1987, he received the Best Paper Award of the IEE Fifth International Conference on Antennas and Propagation. In 1994, he received the IEEE APS Best Application Paper Award (H. A. Wheeler Award). He is an Associate Editor of IEEE Antennas and Propagation Magazine. 